\title{
ON THE CALCULATION OF VARIANCES AND CREDIBILITIES BY EXPERIENCE RATING
}

\author{
K. LOIMARANTA \\ Finland
}

\section{INTRODUCTION}

By experience rating the main problem is to estimate the credibilities. We have for the credibility $\alpha_{k}$ the famous formula ${ }^{*}$ )

$$
\alpha_{k}=\frac{\sigma_{0}^{2}}{\sigma_{0}^{2}+\sigma_{k}^{2}}
$$

but it is often troublesome to find suitable estimates for the variances $\sigma_{0}^{2}$ and $\sigma_{k}^{2}$. In the present paper a general method to estimate them from the actual statistics is given.

A disadvantage of the method is that good estimates require relatively extensive statistical material. If one of the variances is known, the method can be easily modified to give the other variance from statistics of moderate size.

The method is based on the Maximum Likelihood principle and leads to a system of non-linear equations. The equations can be solved by an iterative process, easily programmable for computers.

The mathematical model underlying the experience rating problem differs in our case lightly from the usual one.

\section{Formulation of the Problem}

We consider a portfolio, which is divided into $N$ classes. In each class we have observed a claim amount per risk unit. Our assumption is that the relative claim amount $y_{k}$ in the class $k(k=\mathrm{I}, 2$, $\ldots, N)$ has a definite but unknown meanvalue $m_{k}$ and a variance $\sigma_{k}^{2}$, which is inverse proportional to some known measure $t_{k}$ of the size of that class, e.g. the number of risk units in the class. We can thus write

$$
\begin{aligned}
& \text { Mean: } \quad E\left(y_{k}\right)=m_{k} \\
& \text { Variance: } V\left(y_{k}\right)=\sigma_{k}^{2}=h / t_{k} \text {. }
\end{aligned}
$$

* Bühlman, H.: Mathematical Methods in Risk Theory. 
As a second step we assume, that the quantities $m_{k}$ are random variables with a common probability distribution. Let this distribution have the meanvalue $m_{0}$ and the variance $\sigma^{2}$ :

$$
\begin{aligned}
& \text { Mean: } \quad E\left(m_{k}\right)=m_{0} \\
& \text { Variance: } V\left(m_{k}\right)=\sigma_{0}^{2} .
\end{aligned}
$$

Assuming that both steps are independent on each other (or at least uncorrelated) we have for the compound random variable $y_{k}$

$$
\begin{array}{ll}
\text { Mean: } \quad E_{c}\left(y_{k}\right) & =m_{0} \\
\text { Variance: } & V_{c}\left(y_{k}\right)=\sigma_{0}^{2}+h / t_{k} .
\end{array}
$$

The result might be better known from the theory of compound Poisson processes.

To calculate the credibilities

$$
\begin{aligned}
\alpha_{k} & =\frac{\sigma_{0}^{2}}{\sigma_{0}^{2}+\sigma_{k}^{2}}=\frac{\sigma_{0}^{2}}{\sigma_{0}^{2}+h / t_{k}} \\
\mathrm{I}-\alpha_{k} & =\frac{\sigma_{k}^{2}}{\sigma_{0}^{2}+\sigma_{k}^{2}}=\frac{h / t_{k}}{\sigma_{0}^{2}+h / t_{k}}
\end{aligned}
$$

we must have estimates for the variance $\sigma_{0}^{2}$ and the constant $h$, which determines the variances $\sigma_{k}^{2}$.

\section{The Maximum Likelihood Solution}

We suppose from now on, that the random variables $y_{k}$ are with required accuracy normally distributed, i.e. $y_{k}$ has the distribution function

$$
f\left(y_{k}\right)=\frac{I}{\sqrt{2 \pi\left(\sigma_{0}^{2}+h / t_{k}\right)}} e^{-\frac{\left(y_{k}-m_{0}\right)^{2}}{2\left(\sigma_{0}^{2}+h / t_{k}\right)}}
$$

We use the Maximum Likelihood method $*$ ) to estimate the parameters $m_{0}, \sigma_{0}^{2}$ and $h$ in the distribution function of $y_{k}$.

For the logarithm of the Likelihood function $L$ we have the expression

$$
\log L=-\sum_{k}\left[\frac{\left(y_{k}-m_{0}\right)^{2}}{2\left(\sigma_{0}^{2}+h / t_{k}\right)}+\frac{1}{2} \log \left(\sigma_{0}^{2}+h / t_{k}\right)\right]+\text { const. }
$$

* Eg. Cramér, H., Mathematical Methods of Statistics. 
Its maximum value is a solution of the equations

$$
\begin{aligned}
& \frac{\partial \log L}{\partial m_{0}}=\sum_{k} \frac{y_{k}-m_{0}}{\sigma_{0}^{2}+h / t_{k}}=0 \\
& \frac{\partial \log L}{\partial\left(\sigma_{0}^{2}\right)}=\sum_{k}\left[\frac{\left(y_{k}-m_{0}\right)^{2}}{2\left(\sigma_{0}^{2}+h / t_{k}\right)^{2}}-\frac{\mathrm{I}}{2\left(\sigma_{0}^{2}+h / t_{k}\right)}\right]=0 \\
& \frac{\partial \log L}{\partial h}=\sum_{k}\left[\frac{\left(y_{k}-m_{0}\right)^{2}}{2\left(\sigma_{0}^{2}+h / t_{k}\right)} \cdot \frac{\mathrm{I}}{t_{k}}-\frac{\mathrm{I}}{2\left(\sigma_{0}^{2}+h / t_{k}\right)} \cdot \frac{\mathrm{I}}{t_{k}}\right]=0
\end{aligned}
$$

Multiplying the first equation by $\sigma_{0}^{2}$, the second by $\sigma_{0}^{4}$ and the last one by $h^{2}$ and 'observing the expression (A) for $\alpha_{k}$ we get the following equations

I)

2)

3)

$$
\begin{aligned}
& \sum_{k} \alpha_{k} y_{k}=m_{0} \sum_{k} \alpha_{k} \\
& \sum_{k} \alpha_{k}\left(y_{k}-m_{0}\right)^{2}=\sigma_{0}^{2} \sum_{k} \alpha_{k} \\
& \sum t_{k}\left(\mathrm{I}-\alpha_{k}\right)^{2}\left(y_{k}-m_{0}\right)^{2}=h \cdot \Sigma\left(\mathrm{I}-\alpha_{k}\right) .
\end{aligned}
$$

From the equations (B) and the expression (A) for $\alpha_{k}$ the quantities $m_{0}, \sigma_{0}^{2}$ and $h$ as well as the credibilities $\alpha_{k}$ can be calculated by an iterative process. We start with arbitrary values for the quantities $\alpha_{k}$ (e.g. $\alpha_{k}=\mathrm{I} / 2$ ) and calculate $m_{0}, \sigma_{0}^{2}$ and $h$ from eq. (B). New values for $\alpha_{k}$ will then be calculated from eq. (A) with the received values of $\sigma_{0}^{2}$ and $h$. Subsequently the new values of $\alpha_{k}$ will be inserted in eq. (B), and so on.

According to our experience about ten steps are required to get the values of $\alpha_{k}$ with an accuracy of o.oor. The method is cumbersome for manual calculation but suits well for electronic computers.

When the credibilities $\alpha_{k}$ are determined the premiums net of charges for different classes can be calculated by the normal way

$$
P_{k}=\alpha_{k} y_{k}+\left(I-\alpha_{k}\right) m_{0} \text {. }
$$

\section{A Possible Generalisation}

The method can be generalized to solve more complicated problems. So far we have assumed the quantitities $m_{k}$ to be drawn from one and the same probability distribution. But we can also think them to be results of a regression analysis

$$
m_{k}=a+\sum_{i} b_{i k}
$$


where $x_{i k}$ is the value of the $i:$ th independent variable in class $k$. In stead of the simple weighted mean

$$
m_{0}=\frac{\sum_{k} \alpha_{k} y_{k}}{\sum_{k} \alpha_{k}}
$$

we have for each step to solve $a$ by $\alpha_{k}$ weighted regression analysis problem and to put in the equations 2) and 3) the residuals in stead of the quantities $m_{k}-m_{0}$.

\section{Discussion of the Method}

The maximum likelihood method is normally used for observations $y_{k}$ with equal distributions and gives then under general assumptions asymptotically optimal estimates. The restriction to equal distributions is unessential but nevertheless we have to be careful. An other point to be observed is that we have assumed the quantities $y_{k}$ to be normally distributed.

The first eq. (B) is same as Hovinen *) has got with a different method in the case $\sigma_{0}^{2}$ and $h$ are given. The equation gives an unbiased minimum variance estimate for the mean independent of the normality of the quantities $y_{k}$. Conserning this equation we are thus on the safe side.

It is interesting to observe that in the credibility theory an other formula is in general used to calculate the gross mean

$$
m_{0}^{\prime}=\frac{\sum t_{k} y_{k}}{\Sigma t_{k}}
$$

The first formula in (B) gives the correct estimate for the mean if we choose one class at random, the formula ( $\left.I^{\prime}\right)$ if we choose one risk unit (policy) at random. The differences between $m_{0}$ and $m_{0}^{\prime}$ can be considerable.

Wore caution is required by the use of the eq. 2) and 3) in (B). It is not enough that the number of observations (classes) $N$ is sufficiently large. If all quantities $t_{k}$ are identical the eq. 2) and 3 ) are linearly dependent and all values $g_{k}=$ const. are a solution of

* Hovinen, E., On the Estimation of Means and Variances in the Case of Unequal Components, ASTIS Bulletin, Vol. VIII, Part 3. 
the equationsystem (A) and (B). An acceptable splitting of the variance

$$
\sigma_{0}^{2}+h / t_{k}
$$

into its components requires thus that the variation of $t_{k}$ is great enough. Is this not the case but one of the components is known, the method can be used to calculate the remaining component and the credibilities $\alpha_{k}$ simply by omitting the corresponding equation in (B).

It is well-known that Maximum Likelihood method gives for finite samples too low values for variances. The bias is normally of the order $\mathrm{r} / N$. An unbiased expression for the variance in the $\operatorname{case}_{0}^{2} \sigma=0$, i.e. $\alpha_{k}=0$ is given by Hovinen (ibid.). His formula (26) is by our notations

$$
h=\frac{\mathrm{I}}{N} \sum_{k} \frac{t_{k}}{\mathrm{I}-\frac{t_{k}}{\Sigma t_{k}}}\left(y_{k}-m_{0}\right)^{2}
$$

Our eq. 3) in (B) gives with $\alpha_{k}=0$

$$
h=\mathrm{I} / N \sum_{k} t_{k}\left(y_{k}-m_{0}\right)^{2}
$$

Comparing these two formulas we see, that the bias is negligible if all $t_{k}:$ are small compared with $\Sigma t_{k}$. 